\title{
EFFETS DE L'HEXADÉCANOL INCORPORÉ A L'EAU D'ÉLEVAGE SUR LA GAMÉTOGENĖSE, LES NIVEAUX GONADOTROPES HYPOPHYSAIRES ET PLASMATIQUES, ET LA CROISSANCE DU POISSON ROUGE
}

\author{
C. GILLET, R. BILLARD \\ INRA, Laboratoire de Physiologie des Poissons \\ 78350 JOUY-EN-JOSAS, France
}

\section{INTRODUCTION}

Des substances chimiques à des concentrations inférieures aux doses léthales peuvent perturber divers aspects de la reproduction. Chez les oiseaux, les pesticides entrainent fréquemment une malformation des gonades et la stérilité des animaux (LUTZ-OSTERTAG et LUTZ, 1974). Chez les poissons, le parathion, de même que le méthyl-mercure peuvent aussi perturber la gamétogenèse et diminuer la fécondité (BILLARD et de KINKELIN, 1970; KIHLSTROM et al., 1971).

Les micropolluants, qui se concentrent au cours de la chaine alimentaire, s'accumulent parfois en quantités très importantes dans les ovaires de poissons prédateurs. Les cufs de Salmonidés arrivent à accumuler des teneurs en organochlorés si importantes que la plupart des embryons issus de ces œufs avortent au début de leur développement (JENSEN et al., 1970).

Ces constatations confirment l'idée, actuellement fort répandue, selon laquelle l'évaluation de la dose léthale $(50 \%)$ n'est pas un critère suffisant pour rendre compte des dommages que peuvent causer les polluants des eaux sur les poissons. La gamétogenèse doit constituer l'un des critères complémentaires dont 
il convient de tenir compte dans la définition des concentrations en micropolluants tolérables dans les eaux.

Dans l'expérience présente, nous avons testé l'effet des alcools gras sur la gamétogenèse d'un Cyprinidé, le poisson rouge.

\section{EXPERIENCE I}

\section{EFFETS DE L'HEXADECANOL SUR LA REPRODUCTION DU POISSON ROUGE (CARASSIUS AURATUS L.)}

\section{Matériel animal et conditions d'élevage}

Des poissons rouges adultes (Carassius auratus) provenant des étangs des pêcheries de Camargue ont été utilisés pour cette expérience. Ces Cyprinidés, voisins de la carpe, très robustes et de petite talle, conviennent parfaitement pour les études en laboratoire. Quatre-vingt-dix de ces animaux, pesant $50 \mathrm{~g}$ environ, sont répartis en trois groupes ( 15 femelles et 15 mâles par lot) et placés dans les bassins contenant les différentes concentrations d'hexadécanol étudiées.

Ces animaux sont nourris quotidiennement avec des granulés "Aqualim" (poissons d'étang). L'oxygénation de l'eau est assuree par un apport permanent d'air comprimé. La température de l'eau des élevages est continuellement enregistrée avec des thermographes Richard.

\section{Protocole expérimental}

Des poissons rouges sont exposes durant trois mois, du 12 mars au 8 juin à deux concentrations d'hexadécanol. Quotidiennement, 0,5 et $0,05 \mathrm{ppm}$ de ce produit sont mélangés à l'eau de deux bassins d'èlevage. Un troisième élevage sert de témoin, sans hexadécanol. Comme cet alcool gras, présenté sous forme de petites paillettes, se dissout très lentement dans l'eau, il est d'abord broyé à lultra turrax, à $200 \mathrm{ppm}$ dans de l'eau distilleee. Une certaine quantité de cette suspension est ensuite déversée dans l'eau des élevages de carassins, pour aboutir à la concentration d'hexadécanol préalablement choisie.

Ce traitement est répété 5 jours par semaine, du lundi au vendredi. Au début de chaque semaine, l'eau des élevages est entièrement renouvelée.

\section{PARAMETRES ETUDIES}

\section{A - L'hormone gonadotrope c-GTH}

\section{1) Les teneurs plasmatiques}

Des échantillons sanguins sont collectés par ponction cardiaque, au début de l'expérimentation, puis le 29-3, le 14-4, le 7-5, le 15-5 et le 1-6. Le sang est recueilli sur héparine, il est ensuite centrifugé $15 \mathrm{mn}$ à $3000 \mathrm{t} / \mathrm{mn}$. Le plasma est conservé à $-20 \circ$ C. Après décongélation, l'hormone gonadotrope plasmatique est dosée par radioimmunologie d'après la méthode de BRETON et al. (1971)

\section{2) Les teneurs hypophysaires}

A l'issue d'abattages, le 12 mars, le 15 avril et le 8 juin, l'hypophyse des animaux sacrifiés est pesée à $\pm 10 \mu \mathrm{g}$ sur une balance Mettler $\mathrm{ME}^{22}$, puis broyée 
finement avec un poter et diluée à $1 \mathrm{~m} / \mathrm{mg}$ dans le tampon du dosage radioimmunologique. Après une dilution à $1 / 2000$, la charge gonadotrope hypophysaire en c-GTH est mesurée par radioimmunologie.

\section{B - Le rapport gonadosomatique (RGS)}

A l'issue des abattages, l'état d'avancement de la gamétogenèse est évalue par le rapport gonadosomatique, défini par la formule



\section{C - Autres paramètres étudiès}

La croissance des animaux est appréciée par le gain pondéral évalué en pourcentage du poids initial et par le coefficient de condition défini comme le

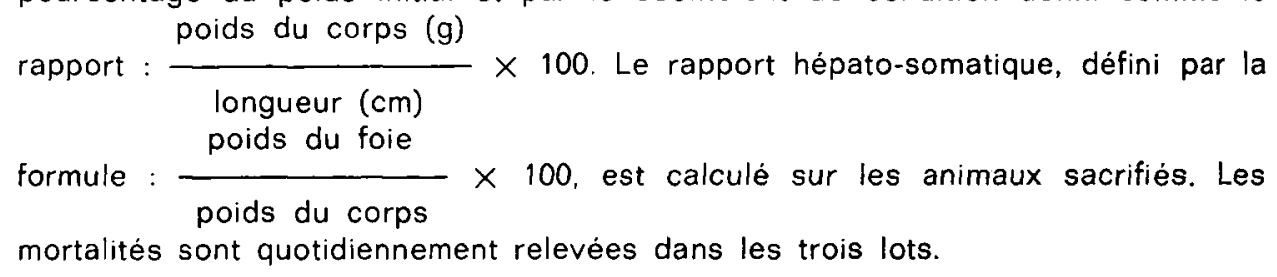
poids du corps (g)

rapport : $\frac{\text { longueur }(\mathrm{cm})}{\text { loids du }} \times 100$. Le rapport hépato-somatique, défini par la formule : $\frac{\text { poids du foie }}{\text { poids du corps }} \times 100$, est calculé sur les animaux sacrifiés. Les

mortalités sont quotidiennement relevées dans les trois lots.

\section{RESULTATS}

\section{A - Effets de l'alcool gras sur les teneurs en hormone gonadotrope}

1) L'hormone gonadotrope plasmatique (fig. 1, 2)

Dans tous les cas, le niveau d'hormone gonadotrope plasmatique augmente à la fin de l'expérience, au mois de juin, lorsque la température de l'eau s'élève. Il existe une relation linéaire entre la concentration en c-GTH plasmatique et la température de l'eau

Par contre, aussi bien chez les femelles que chez les mâles, les différentes concentrations d'hexadécanol ne provoquent aucune variation significative du taux d'hormone gonadotrope, même après trois mois d'exposition (tableau 1).

Tableau 1 : Comparaison par analyse de variance des teneurs plasmatiques en $c-G T H$ entre les trois lots ( $T, F, F)$

\begin{tabular}{|c|c|c|c|c|}
\hline \multirow{2}{*}{$\begin{array}{c}\text { Date de } \\
\text { prélèvement }\end{array}$} & \multicolumn{2}{|c|}{$0^{\nearrow}$} & \multicolumn{2}{|c|}{$\stackrel{O}{+}$} \\
\hline & Facteur $F$ & d. 1 & Facteur F & d.I \\
\hline $\begin{array}{r}29-03 \\
14-04 \\
7-05 \\
15-05 \\
1-06\end{array}$ & $\begin{array}{l}2,686 \\
0,220 \\
0,002 \\
0,824 \\
1,288\end{array}$ & $\begin{array}{l}2 / 12 \\
2 / 32 \\
2 / 19 \\
2 / 19 \\
2 / 18\end{array}$ & $\begin{array}{l}7,891 \\
0,111 \\
0,532 \\
0,809 \\
0,630\end{array}$ & $\begin{array}{l}2 / 25 \\
2 / 29 \\
2 / 25 \\
2 / 18 \\
2 / 15\end{array}$ \\
\hline
\end{tabular}




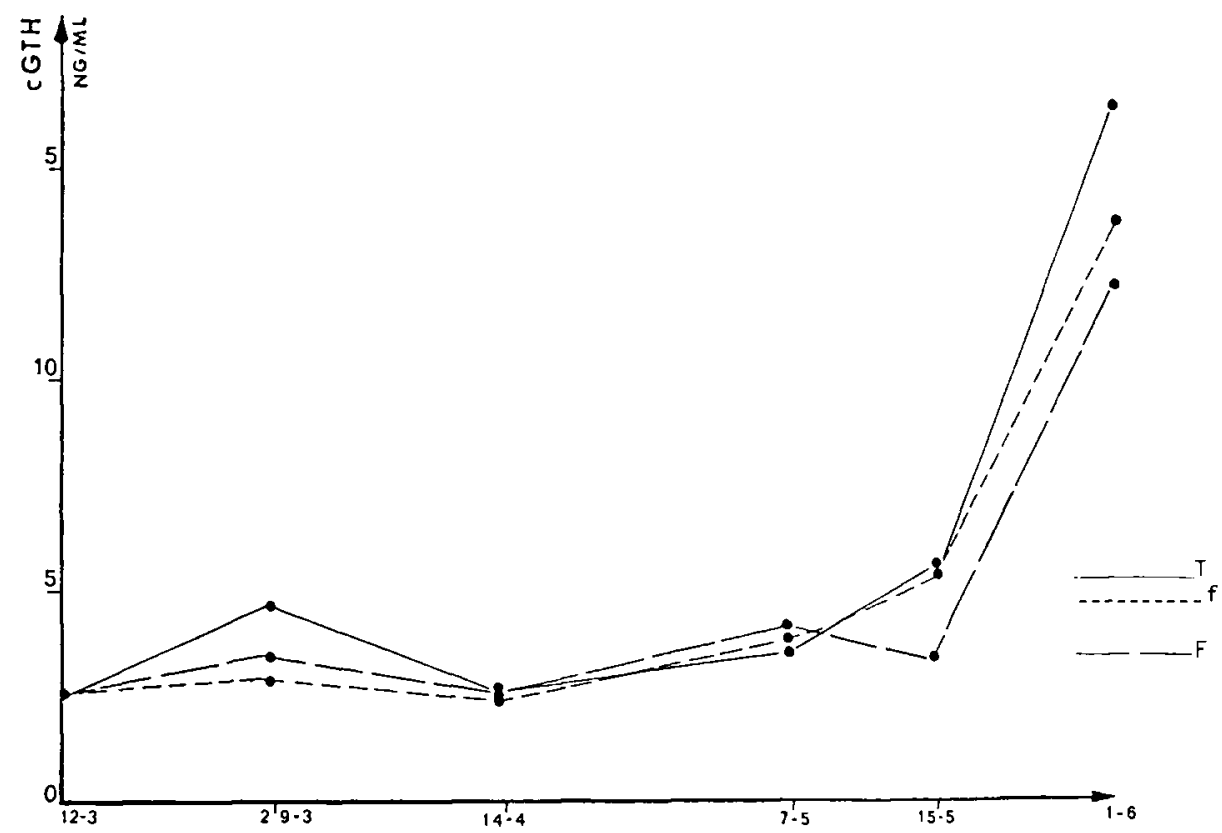

Fig. 1 : Effets de l'hexadécanol sur les teneurs plasmatiques en hormone gonadotrope (c-GTH) chez le poisson rouge femelle. $T$ : témoins, $f$ : dose faible, $F$ : dose forte.

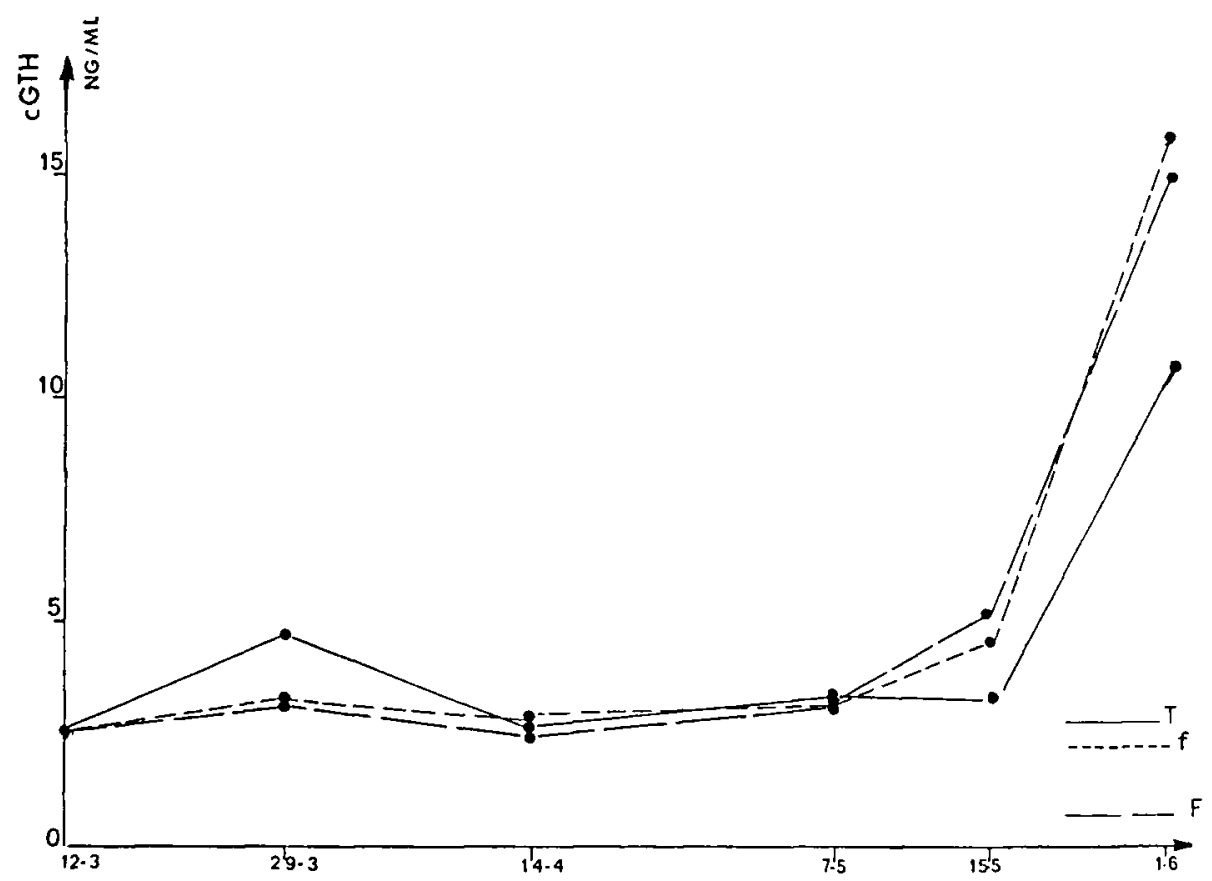

Fig. 2 : Effets de l'hexadécanol sur les teneurs plasmatiques en hormone gonadotrope chez le poisson rouge mâle. 
2) L'hormone gonadotrope hypophysaire (fig. 3)

Chez les femelles comme chez les màles, la charge gonadotrope hypophysaire n'est pas modifiée par l'apport d'hexadécanol. quelle que soit la durée de l'exposition (tableau 2).

Tableau 2 : Analyse de variance sur la charge en c-GTH hypophysaire entre les trois élevages $(T, f, F)$

\begin{tabular}{|c|c|c|c|c|}
\hline \multirow{2}{*}{$\begin{array}{c}\text { Date de } \\
\text { prèlèvement }\end{array}$} & \multicolumn{2}{|c|}{$0^{\nearrow}$} & \multicolumn{2}{|c|}{$\begin{array}{l}0 \\
+ \\
\end{array}$} \\
\hline & Facteur $F$ & d.I & Facteur $\mathrm{F}$ & d.I \\
\hline $\begin{array}{r}14-04 \\
8-06\end{array}$ & $\begin{array}{l}0,303 \\
0,644\end{array}$ & $\begin{array}{l}2 / 12 \\
2 / 17\end{array}$ & $\begin{array}{l}0,097 \\
2,899\end{array}$ & $\begin{array}{l}2 / 11 \\
2 / 14\end{array}$ \\
\hline
\end{tabular}
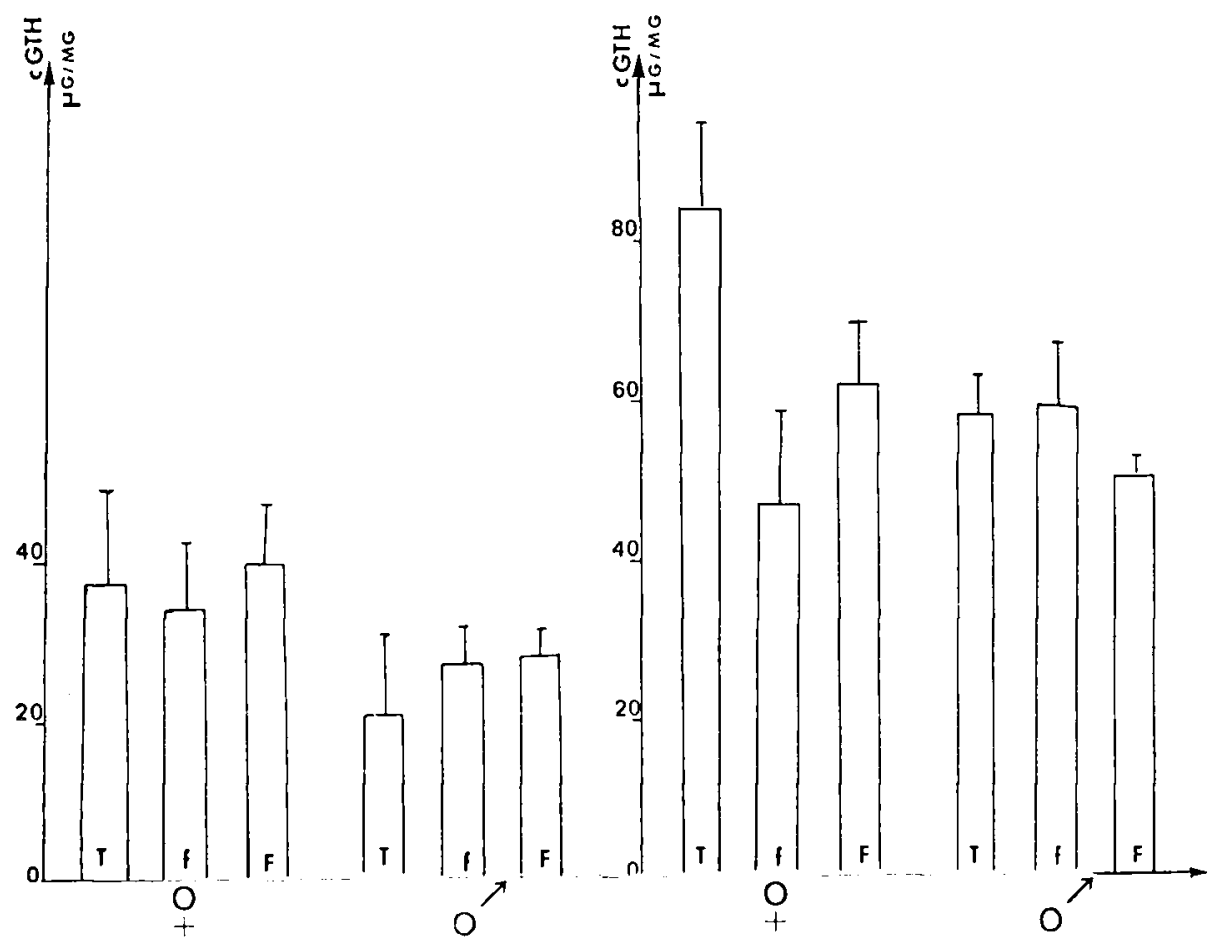

Fig. 3 : Effets de l'hexadécanol sur les teneurs hypophysaires en hormone gonadotrope chez le poisson rouge (mâle et femelle) : à gauche : 14-4; à droite : 8 - 6 .

B - Effet de l'hexadécanol sur la gamétogenèse (fig. 4)

1) Les femelles

Les ovaires sont déjà très développés au début de l'expérimentation, le 12-03. Les ovocytes sont chargés de réserves vitellines. L'exposition des femelles 


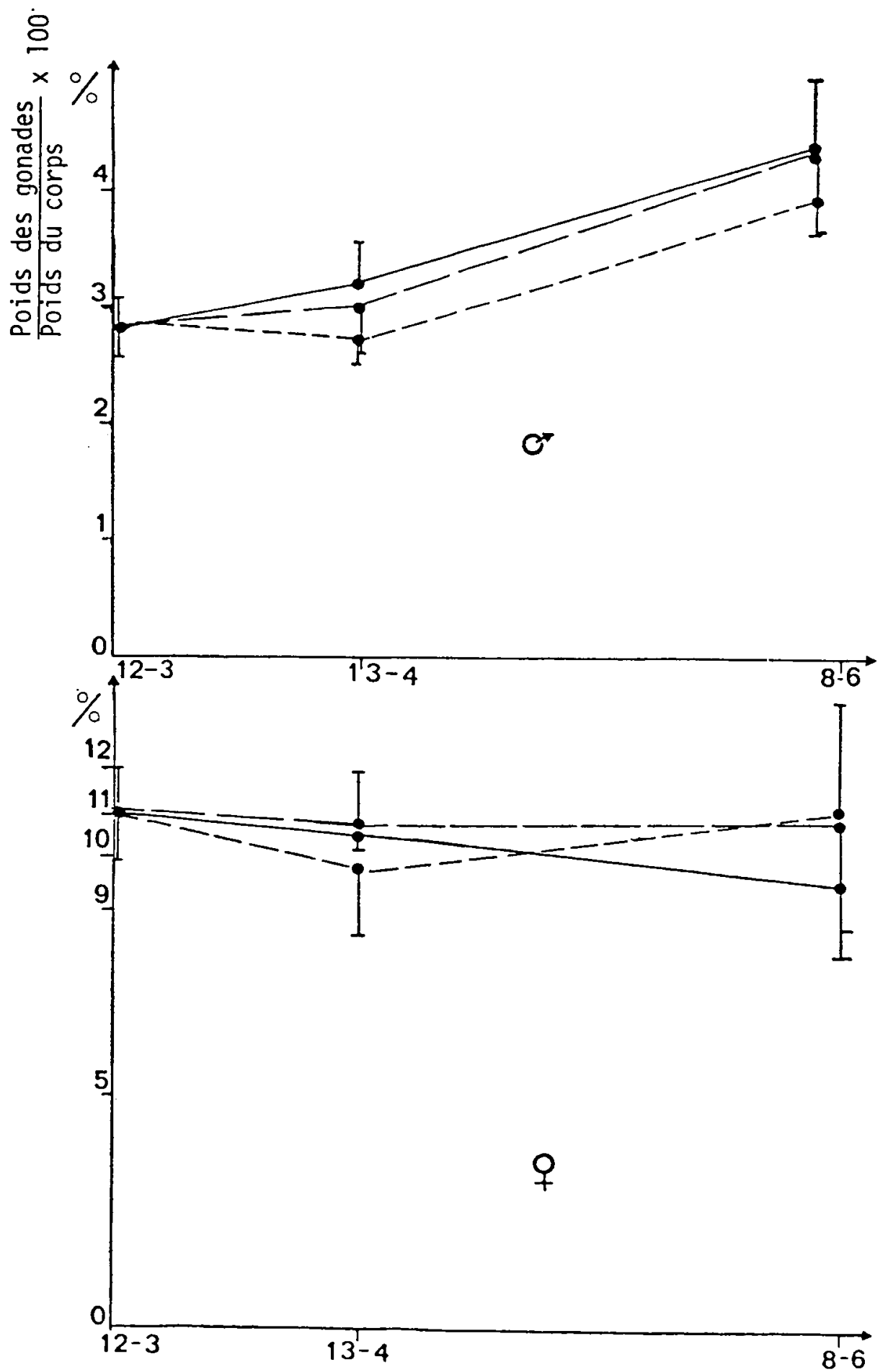

Fig. 4 : Effet de l'hexadécanol sur les rapports gonadosomatiques (RGS) des poissons rouges (mâles et femelles). 
aux diffèrentes concentrations d'hexadécanol ne perturbe pas l'achèvement de la vitellogenèse. Les valeurs du rapport gonadosomatique restent constantes, quelle que soit la concentration en alcool gras des lots experimentaux au cours des trois mois que dure l'exposition (tableau 3)

\section{2) Les mâles}

Dans tous les cas, le volume testiculaire s'accroit lentement de mars à juin. L'analyse statistique ne permet pas de déceler de différences significatives en fonction de la concentration d'hexadécanol de l'élevage ni en avril, ni en juin (tableau 3)

Tableau 3 : Analyse de variance sur les RGS des poissons des trois lots (T, $f, F)$

\begin{tabular}{|c|c|c|c|c|}
\hline \multirow{2}{*}{$\begin{array}{c}\text { Date de } \\
\text { prélèvement }\end{array}$} & \multicolumn{2}{|c|}{$0^{\nearrow}$} & \multicolumn{2}{|c|}{$\begin{array}{l}0 \\
+\end{array}$} \\
\hline & Facteur F & d.I & Facteur F & d.l \\
\hline $\begin{array}{r}14-04 \\
8-06\end{array}$ & $\begin{array}{l}0,757 \\
0,693\end{array}$ & $\begin{array}{l}2 / 14 \\
2 / 18\end{array}$ & $\begin{array}{l}0,518 \\
0,202\end{array}$ & $\begin{array}{l}2 / 11 \\
2 / 14\end{array}$ \\
\hline
\end{tabular}

\section{Effet de l'hexadécanol sur le RHS et la croissance}

1) Le rapport hépatosomatique (RHS) (fig. 5)

Au cours du premier mois d'expérimentation, la valeur du rapport hépatosomatique des animaux s'accroit dans tous les élevages $(P<0,05)$. Puis, ces valeurs du RHS diminuent lorsque la température de l'eau s'élève, entre le 14-04 et le 8-06 $(P<0,05)$.

Par contre, pour les mâles comme pour les femelles, l'analyse statistique ne permet pas de mettre en évidence de différences significatives en fonction du traitement à l'hexadécanol (tableau 4).

Tableau 4 : Comparaison par analyse de variance des RHS des animaux des élevages $T, f, F$

\begin{tabular}{|c|c|c|c|c|}
\hline \multirow{2}{*}{$\begin{array}{c}\text { Date de } \\
\text { prélèvement }\end{array}$} & \multicolumn{2}{|c|}{$0^{\nearrow}$} & \multicolumn{2}{|c|}{$\begin{array}{l}0 \\
+\end{array}$} \\
\hline & Facteur $F$ & d.I & Facteur F & d.l \\
\hline $\begin{array}{r}14-04 \\
8-06\end{array}$ & $\begin{array}{l}0,137 \\
0,275\end{array}$ & $\begin{array}{l}2 / 13 \\
2 / 17\end{array}$ & $\begin{array}{l}0,243 \\
0,135\end{array}$ & $\begin{array}{l}2 / 11 \\
2 / 13\end{array}$ \\
\hline
\end{tabular}

2) Le coefficient de condition (fig. 6)

Pour les animaux des deux sexes, en avril comme en juin, les différentes concentrations d'hexadécanol ne provoquent pas de variations significatives du coefficient de condition (tableau 5) 


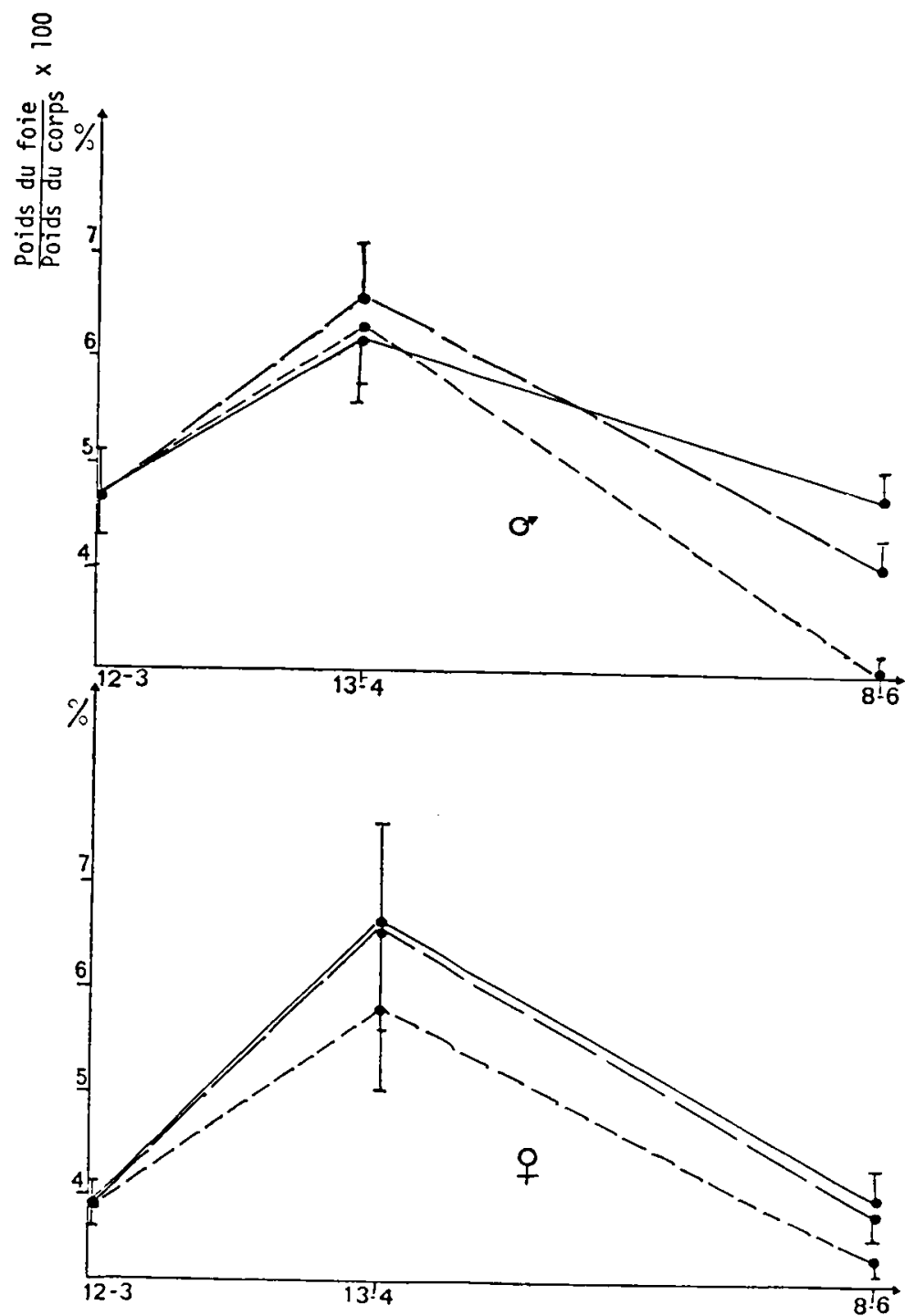

Fig. 5 : Effet de l'hexadécanol sur les rapports hépatosomatiques (RHS) des poissons rouges (mâles et femelles). 
Tableau 5 : Comparaison par analyse de variance entre les coefficients de condition $(T, f, F)$

\begin{tabular}{|c|c|c|c|c|}
\hline \multirow{2}{*}{$\begin{array}{c}\text { Date de } \\
\text { prélèvement }\end{array}$} & \multicolumn{2}{|c|}{$0^{\nearrow}$} & \multicolumn{2}{|c|}{+} \\
\hline & Facteur $F$ & d.I & Facteur $\mathrm{F}$ & d.I \\
\hline $\begin{array}{r}14-04 \\
8-06\end{array}$ & $\begin{array}{l}0,065 \\
0,321\end{array}$ & $\begin{array}{l}2 / 13 \\
2 / 18\end{array}$ & $\begin{array}{l}2,124 \\
1,161\end{array}$ & $\begin{array}{l}2 / 21 \\
2 / 13\end{array}$ \\
\hline
\end{tabular}

3) La croissance (fig. 7)

Bien qu'il n'y ait jamais de différences significatives au seuil de $5 \%$ entre les différents lots, la croissance des animaux exposés à l'hexadécanol est toujours plus faible que celle des témoins, pour les mâles comme pour les femelles (tableau 6).

Tableau 6 : Analyse de variance entre les taux de croissance des poissons des trois lots $(T, F, F)$

\begin{tabular}{|c|c|c|c|c|}
\hline \multirow{2}{*}{$\begin{array}{c}\text { Date de } \\
\text { prélèvement }\end{array}$} & \multicolumn{2}{|c|}{$0^{7}$} & \multicolumn{2}{|c|}{$\begin{array}{l}0 \\
+ \\
\end{array}$} \\
\hline & Facteur $\mathrm{F}$ & d.I & Facteur $\mathrm{F}$ & d.I \\
\hline $\begin{array}{r}14-04 \\
8-06\end{array}$ & $\begin{array}{l}0,224 \\
2,342\end{array}$ & $\begin{array}{l}2 / 14 \\
2 / 13\end{array}$ & $\begin{array}{l}2,270 \\
2,230\end{array}$ & $\begin{array}{l}2 / 11 \\
2 / 10\end{array}$ \\
\hline
\end{tabular}

D - La mortalité (tableau 7)

Les mortalités semblent avoir été provoquées par des maladies bactériennes (furonculose) et par des champignons parasites (saproléniose).

Tableau 7 : Mortalités cumulées du $12-04$ au 8-06

\begin{tabular}{|c|c|c|}
\hline $\begin{array}{c}\text { Elevage témoin } \\
(T)\end{array}$ & Dose faible & Dose forte \\
$(f)$ & $(F)$ \\
$20 \%$ & $0 \%$ & $20 \%$ \\
\hline
\end{tabular}

Test du $X^{2}=5,99$ (degrés de liberté : 2)

Le test statistique indique que la différence observée est à la limite du seuil de $5 \%$. Cependant, ies mortalites enregistrees pour les poissons exposés à la plus forte dose d'hexadécanol sont les mêmes que celles enregistrées dans l'élevage témoin. 


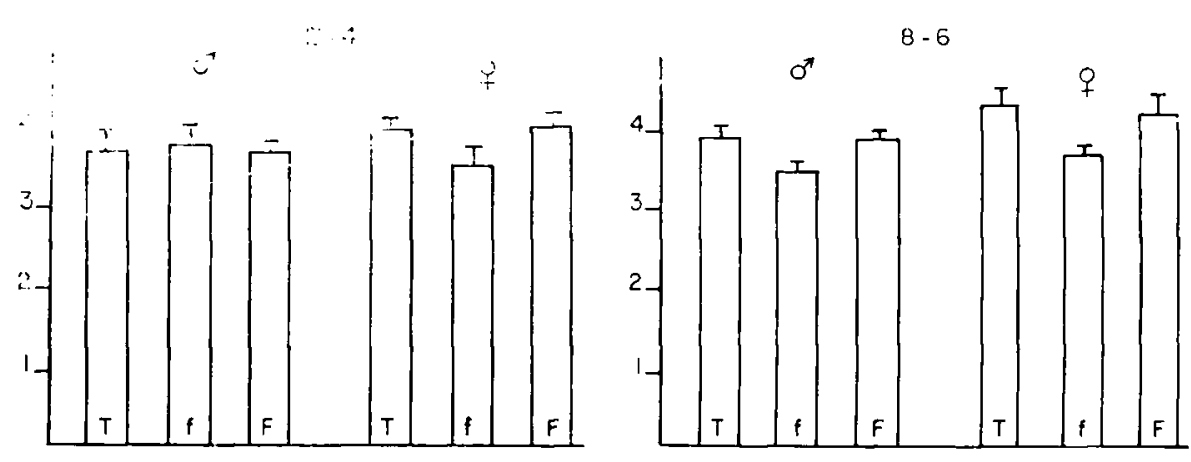

Fig. 6 : Effet de l'hexadécanol sur les coefficients de condition $(K)$ des poissons rouges (mâles et femelles). $K=\left[\right.$ Poids / Longueur) $\left.{ }^{2}\right] \times 100$.
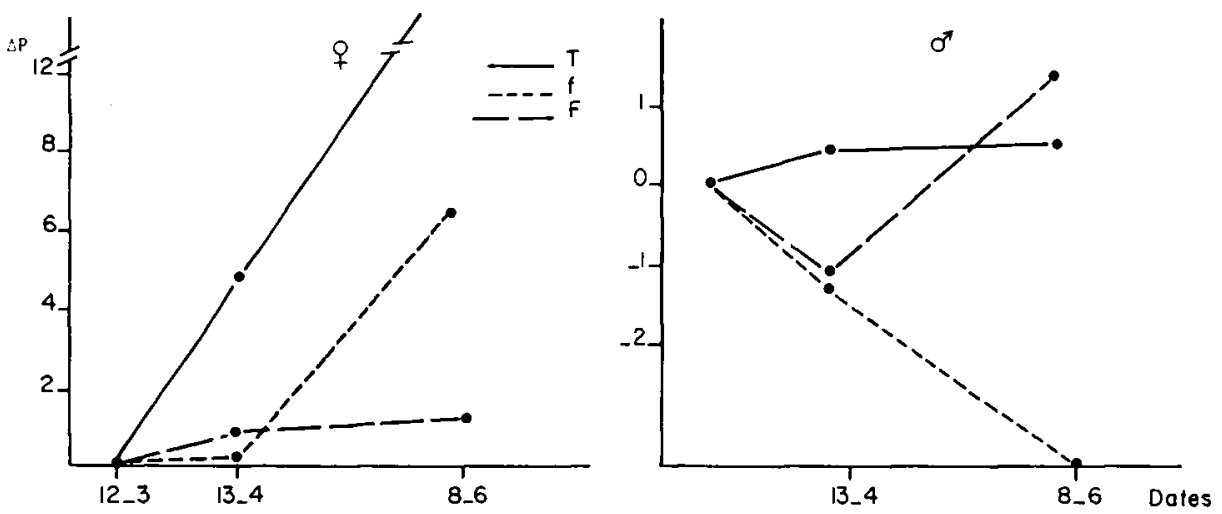

Fig. 7 : Effet de l'hexadécanol sur le taux de croissance des poissons rouges (mâles et femelles). $\lrcorner P=[$ Poids final / Poids initial $] \times 100$. 


\section{DISCUSSION ET CONCLUSIONS}

Dans nos conditions expérimentales, nous n'avons observé aucun effet de l'hexadécanol, ni sur la sécrétion gonadotrope, ni sur le déroulement de la gamétogenèse. Cependant, d'autres étapes de la reproduction n'ont pas pu être testees. Par exemple, l'ovulation qui ne se produit pas spontanément en captivité chez le poisson rouge. II serait intéressant de connaitre les effets des alcools gras sur l'ovulation et sur le comportement de ponte de ces animaux, car des substances chimiques dans l'eau, les phéromones, interviennent au cours de ces étapes chez le poisson rouge (STACEY et LILEY, 1974). Chez une autre espèce, le Brachydanio rerio, la quantitè d'œufs déposés par la femelle est beaucoup plus faible en présence d'acètate de mercure (KIHLSTROM et al., 1971).

D'autre part, la présence de gamètes apparemment normaux ne constitue pas un critere suffisant pour conclure à l'absence d'effet d'un polluant sur la fonction de reproduction. En effet, des mortalités embryonnaires très importantes ont été observèes chez piusieurs espèces de Salmonidés, à la suite d'une accumulation de pesticides organochlorés dans les réserves vitellines de l'œuf (MACEK, 1968 a et b; HOPKINS, 1969 ; LOCKE, 1972). Il reste donc à s'assurer que la fertilité des gamètes ne soit pas altérée par des concentrations excessives d'hexadécanol, en particulier dans l'œuf.

Bien que la diminution du rythme de croissance des poissons rouges sous l'effet de l'hexadécanol soit inférieure au seuil de sensibilité de $5 \%$ du test statistique, nous ne pouvons pas exclure une action défavorable de ce produit sur la croissance. Des modifications de croissance, consécutives à l'administration d'un polluant, ont déjà été rapportées (MACEK, 1968 b).

II faut aussi remarquer que toutes ces expériences ont été effectuées à des températures relativement basses (de 3 à $20 \circ \mathrm{C}$ dans les élevages des poissons rouges). Or, il a été démontré que les températures élevées peuvent potentialiser les effets d'un polluant (GILLET, données non publiées).

Malgré ces réserves portant sur la nécessité d'étendre les recherches à d'autres étapes du cycle ou de les réaliser dans des conditions d'environnement différentes, il apparait que les alcools gras mis dans le milieu aqueux n'affectent pas, de façon notable, les processus de reproduction à des doses inférieures à $0,5 \mathrm{ppm}$ dans nos conditions expérimentales.

\section{REMERCIEMENTS}

Ce travail a été partiellement financé par la Météorologie Nationale (contrat no 65.1851). Nous remercions Madame RIGAUD qui a mis à notre disposition l'hexadécanol utilisé dans cette expérience.

\section{RESUME}

Des doses d'hexadécanol de 0,5 à $0.05 \mathrm{ppm}$ incorporées quotidiennement à l'eau d'élevage ne modifient pas les teneurs en gonadotropine (c-GTH) plasmatique et hypophysaire du poisson rouge par rapport au lot témoin. De même, le rapport gonadosomatique (RGS), le rapport hépatosomatique (RHS), le coefficient de condition et la mortalité ne sont pas modifiés après une durée de traitement de 3 mois. Par contre, la croissance des animaux ayant reçu le traitement ì l'hexadécanol a subi une légère dépression par rapport aux animaux témoins, mais les différences ne sont pas significatives. 


\section{SUMMARY}

Hexadecanol at doses of 0.5 and 0.05 ppm incorporated every day to the water did not modify plasma and pituitary gonadotropin (c-GTH) contents in goldfish when compared to the controls. Similarly gonadosomatic index (GSI), hepatosomatic index (HSI), coefficient of condition and mortality were not changed following 3 month treatment. However, the growth rate of hexadecanol treated animals was slightly depressed as compared to the controls, but differences are not significant.

\section{REFERENCES}

BILLARD R., de KINKELIN P., 1970. Sterilisation des testicules de Guppies par des doses non léthales de parathion. Ann. Hydrobiol., 1, 91-99.

BRETON B., KANN G., BURZAWA-GERARD E., BILLARD R., 1971. Dosage radioimmunologique d'une hormone gonadotrope de Carpe Cyprinus carpio L. C. R. Acad. sci. Paris, 272. 1 515-1517.

JENSEN S., JOHANSSON N., OLSSON M., 1970. PCB indications of effects on salmon. Swedish Salmon Research Institute. Report L.F.I. MEDD 7.

HOPKINS C.K., SALLY S.R.B., RITCHIE A.R., 1969. DDT in trout and its possible effect on reproductive potential. N.Z. Journal of Marine and Freshwater Research. Vol. 3, no 2, June 1969.

KIHLSTROM J.E., LANDERY C., HULTH L., 1971. Number of eggs and young produced by Zebrafishes (Brachydanio rerio, Ham-Buch). Spawning in water containing small amounts of Phenylmercuric acetate. Env. Res., 4, 355-359.

LOCKE D.O., HAVEY K., 1972. Effects of DDT upon Salmon from Schecdic (Maine). Trans. Am. Fish. Soc., 101, 638-643.

LUTZ-OSTERTAG Y., LUTZ H., 1974. Sexualité et pesticides. Ann. Biol. T. XIII, Fasc. 34, 1974.

MACEY K.J., 1968 a. Reproduction in brook trout (Salvelinus fontinalis) fed sublethal concentrations of DDT. J. Fish. Res. Bd. Can., 25, $1787-1796$.

MACEY K.J., 1968 b. Growth and resistance to stress in brook trout fed sublethal levels of DDT. J. Fish. Res. Bd Can., 25, 2 443-2 451.

STACEY N.E., LILEY N.R., 1974. Regulation of spawning behaviour in the female goldfish. Nature, 241, 71. 